\title{
Evaluation de la séroprévalence de la trypanosomose bovine en Guinée
}

\author{
A.M. Barry ${ }^{1 *}$ F. Roger ${ }^{2}$ M.B. Diallo ${ }^{1}$ S. Geerts ${ }^{3}$
}

Mots-clés

Bovin - Antigène - Anticorps Trypanosome - Immunodiagnostic Guinée.

\begin{abstract}
Résumé
Une évaluation de la prévalence de la trypanosomose a été menée en Guinée sur des sérums issus de la sérothèque du laboratoire vétérinaire de la Direction nationale de l'Elevage. Neuf cent vingt-huit sérums provenant de bovins N'dama ont été sélectionnés par randomisation et analysés par le test de détection d'anticorps et d'antigène de Trypanosoma spp. Les résultats obtenus par le premier test ont révélé la présence d'anticorps de Trypanosoma spp. chez 67 p. 100 des bovins, avec des taux de 72 p. 100 dans la région de la Haute Guinée, de 68 p. 100 en Basse Guinée, de 63 p. 100 en Guinée forestière et de 62 p. 100 en Moyenne Guinée. Parmi les animaux, 69 p. 100 des femelles étaient infectées ou avaient été en contact avec l'agent infectieux, contre 62 p. 100 des mâles. Les animaux étaient infectés indépendamment de leur âge. Les proportions d'infections ont été de 71, 43, 61, 63, 59, et 74 p. 100, respectivement chez les bovins âgés de moins d'un an, de 1 à 2 ans, 2 à 3 ans, 3 à 4 ans, 4 à 5 ans, et plus de 5 ans. Le second test a montré la présence d'antigènes de T. brucei (16 p. 100 des sérums), de T. congolense (11 p. 100) et de T. vivax (2 p. 100). La répartition et la combinaison des résultats ont montré que 2 p. 100 des animaux étaient positifs à un, deux, ou trois antigènes sans trace d'anticorps ; 17 p. 100 des animaux ont été positifs aux antigènes et aux anticorps et 50 p. 100 ont eu des anticorps seuls.
\end{abstract}

\section{INTRODUCTION}

Les trypanosomoses animales représentent un problème important en Afrique et dans certaines zones d'Asie et d'Amérique (4). En 2005, la Guinée a défini sa politique de santé publique en élevage. Cette politique vise à connaître la répartition des maladies d'origine parasitaire et à définir leur incidence et leur impact économique. La trypanosomose est une maladie parasitaire, endémique en Guinée, et qui s'inscrit dans cette politique. Les informations sur sa répartition et son importance dans les zones climatiques de la Guinée ne sont pas bien connues. L'outil le plus adapté pour évaluer l'importance des trypanosomoses dans la population bovine est la détection des anticorps dirigés contre les trypanosomes (3). Ce test ne signifie pas que l'animal est porteur de parasites (3). Un test de type Elisa, proposé par Nantulya et Lindqvist, met en évi-

\footnotetext{
1. Direction nationale de l'Elevage, BP 559, Conakry, Guinée. Tél. : + 22460513006 ; e-mail : abarrymadiou@yahoo.fr 2. Cirad, UPR Animal et gestion intégrée des risques, Montpellier, France. 3. Département de santé animale, Institut de médecine tropicale Prince Léopold, Anvers, Belgique.
}

dence les antigènes circulants de Trypanosoma brucei, T. congolense et $T$. vivax (6). Grâce à ce test Elisa, l'importance des espèces de trypanosomes sévissant dans les différentes zones climatiques en Guinée peut être connue.

L'objectif de cette étude a été de connaître la séroprévalence des bovins vivant dans les différentes zones climatiques et d'envisager à terme une meilleure connaissance de l'épidémiologie de la maladie dans les zones à forte prévalence, en vue de mettre sur place une stratégie de contrôle intégré de la maladie.

\section{MATERIEL ET METHODES}

La structure administrative de la Guinée comprend les préfectures, les sous-préfectures, les villages. Dans les villages, les bovins de race N'dama sont regroupés en troupeaux. La taille moyenne du troupeau dans un village est de 50 têtes. La sérothèque du laboratoire vétérinaire de la Direction nationale de l'Elevage a été constituée en utilisant le troupeau comme unité. Dans chaque unité d'échantillons, le tiers des animaux (15 animaux) a fait l'objet d'un prélèvement sanguin en utilisant des tubes secs. 
Après sédimentation des hématies, les sérums obtenus ont été aliquotés dans des cryotubes, puis congelés et conservés à $-20^{\circ} \mathrm{C}$. A chaque utilisation, les échantillons ont été décongelés. Au total, 928 sérums ont été sélectionnés par randomisation, puis analysés par la technique Elisa de capture d'anticorps (5) et la technique Elisa de détection d'antigènes circulants de trypanosomes (6). Les sérums analysés provenaient des quatre régions administratives et éco-climatiques : Basse Guinée, Moyenne Guinée, Haute Guinée, Guinée forestière. La saisie et l'analyse des données ont été effectuées avec le logiciel EPI Info.

\section{RESULTATS}

Les résultats d'analyse des 928 sérums bovins ayant fait l'objet d'une recherche des anticorps anti-trypanosomes et des antigènes de Trypanosoma brucei, $T$. congolense et $T$. vivax sont présentés dans le tableau I.

La prévalence de l'infection à Trypanosoma spp. selon l'âge et le sexe est montrée dans les tableaux II et III.

La combinaison des résultats a montré que 2 p. 100 (21/928) des sérums étaient positifs à un, deux ou trois antigènes sans trace d'anticorps, 17 p. 100 (156/928) des sérums étaient positifs pour les antigène(s) et anticorps, et 50 p. 100 (468/928) des sérums étaient positifs pour des anticorps seuls (tableau IV).

\section{DISCUSSION ET CONCLUSION}

L'étude a révélé que globalement 67 p. 100 des animaux testés avaient des anticorps contre Trypanosoma spp. Ce résultat semble montrer l'endémicité des différentes zones climatiques. Sur le plan immunologique, le caractère trypanotolérant des N'dama s'exprime par une forte réponse en anticorps.

L'âge n'a pas eu d'influence sur l'infection, les animaux s'étant exposés à la maladie à tous les âges. Il y a eu une différence significative entre l'infection chez les males et les femelles $(\mathrm{p}=0,04)$.

Les antigènes circulants ont été mis en évidence chez 16 p. 100 des animaux pour les infections à T. brucei, 11 p. 100 pour les infections à $T$. congolense, 2 p. 100 pour celles à $T$. vivax. Il faut interpréter ces résultats avec prudence parce que le test de détection des antigènes circulants a montré une spécificité et une sensibilité réduites (1). Au Burkina Faso, dans le cadre d'un suivi épidémiologique, les sensibilités du test de détection des antigènes circulants étaient proches de celles relevées lors des infections naturelles chez les bovins (2). Deux pour cent des sérums testés étaient positifs pour les antigènes et négatifs pour les anticorps, ce qui indiquerait que les antigènes sont détectés très tôt avant la production des anticorps. Les infections à $T$. congolense et à $T$. vivax sont détectées 10 à 12 jours après l'infection et celles à $T$. brucei le sont 8 à 14 jours après l'infection (6).

\section{Tableau I}

Prévalence des antigènes circulants et des anticorps de Trypanosoma brucei, $T$. congolense et $T$. vivax dans les sérums de bovins N'dama provenant des quatre régions de la Guinée

\begin{tabular}{|c|c|c|c|c|c|c|c|c|c|c|c|c|c|}
\hline \multirow[t]{2}{*}{ Bovin } & \multirow[t]{2}{*}{ Nb. de sérums } & \multicolumn{3}{|c|}{ T. brucei } & \multicolumn{3}{|c|}{ T. congolense } & \multicolumn{3}{|c|}{ T. vivax } & \multicolumn{3}{|c|}{ Anticorps } \\
\hline & & Pos. & Neg. & $\%$ & Pos. & Neg. & $\%$ & Pos. & Neg. & $\%$ & Pos. & Neg. & $\%$ \\
\hline BG & 337 & 56 & 281 & 17 & 37 & 300 & 11 & 9 & 328 & 3 & 228 & 109 & 68 \\
\hline MG & 242 & 41 & 201 & 17 & 28 & 214 & 12 & 5 & 237 & 2 & 149 & 93 & 62 \\
\hline $\mathrm{HG}$ & 289 & 38 & 251 & 13 & 33 & 256 & 11 & 4 & 285 & 1 & 209 & 80 & 72 \\
\hline GF & 60 & 10 & 50 & 17 & 8 & 52 & 13 & 4 & 56 & 7 & 38 & 22 & 63 \\
\hline Total & 928 & 145 & 783 & 16 & 106 & 822 & 11 & 22 & 906 & 2 & 624 & 304 & 67 \\
\hline
\end{tabular}

BG : Basse Guinée ; MG : Moyenne Guinée ; HG : Haute Guinée ; GF : Guinée forestière

\section{Tableau II}

Prévalence des anticorps de Trypanosoma spp. chez les bovins N'dama selon I'âge

\begin{tabular}{lcccc} 
Age & Nb. de sérums & \multicolumn{3}{c}{ Anticorps } \\
\cline { 3 - 5 } & & Pos. & Neg. & \% \\
\hline$\leq 1$ an & 7 & 5 & 2 & 71 \\
$1-2$ ans & 60 & 26 & 34 & 43 \\
$2-3$ ans & 84 & 51 & 33 & 61 \\
$3-4$ ans & 137 & 86 & 51 & 63 \\
$4-5$ ans & 112 & 66 & 46 & 59 \\
$>5$ ans & 528 & 390 & 138 & 74 \\
Total & $\mathbf{9 2 8}$ & $\mathbf{6 2 4}$ & $\mathbf{3 0 4}$ & $\mathbf{6 7}$
\end{tabular}

\section{Tableau III}

Prévalence des anticorps de Trypanosoma spp. chez les bovins N'dama selon le sexe

\begin{tabular}{lcccc} 
Bovin & Nb. de sérums & \multicolumn{3}{c}{ Anticorps } \\
\cline { 3 - 5 } & & Pos. & Neg. & \% \\
\hline Femelle * $^{*}$ & 655 & 453 & 202 & 69 \\
Mâle * $^{*}$ & 269 & 168 & 101 & 62 \\
Total & $\mathbf{9 2 4}$ & $\mathbf{6 2 1}$ & $\mathbf{3 0 3}$ & $\mathbf{6 7}$ \\
\hline
\end{tabular}

$* \mathrm{P}<0,04$

Sexe non identifié pour quatre sérums 
La trypanosomose existe dans toutes les zones climatiques de la Guinée. La prévalence est importante en Haute Guinée (72 p. 100). Cette zone est réputée pour la production cotonnière, cette dernière faisant recours à la traction animale. Le stress physique peut être à

\section{Tableau IV}

Répartition et combinaison des résultats

\begin{tabular}{|c|c|c|c|c|c|}
\hline & Tb & Tc & Tv & Ac & $\mathrm{Nb}$ \\
\hline & + & - & - & - & 10 \\
\hline & + & + & - & - & 7 \\
\hline & + & - & + & - & 0 \\
\hline & + & - & - & + & 53 \\
\hline & + & + & + & - & 0 \\
\hline & + & - & + & + & 1 \\
\hline & + & + & - & + & 62 \\
\hline & + & + & + & + & 12 \\
\hline & - & + & - & - & 2 \\
\hline & - & - & + & - & 2 \\
\hline & - & - & - & + & 468 \\
\hline & - & + & + & - & 0 \\
\hline & - & + & - & + & 21 \\
\hline & - & - & + & + & 5 \\
\hline & - & + & + & + & 2 \\
\hline & - & - & - & - & 283 \\
\hline Total & & & & & 928 \\
\hline
\end{tabular}

$\mathrm{Tb}:$ Trypanosoma brucei $; \mathrm{Tc}:$ T. congolense $; \mathrm{Tv}:$ T. vivax $; \mathrm{Ac}:$ anticorps l'origine de cette maladie dans les troupeaux N'dama. Il est opportun de connaître la situation épidémiologique de la maladie dans cette zone de production de coton de la Guinée, en vue de mettre en place une stratégie intégrée de contrôle de la maladie.

\section{BIBLIOGRAPHIE}

1. EISLER M.C., PIERRE L., MASAKE R.A., MOLOO S.K., PEREGRINE A.S., 1998. Sensitivity and specificity of antigen-capture ELISAs for diagnosis of Trypanosoma congolense and Trypanosoma vivax infections in cattle. Vet. Parasitol., 79: 187-201.

2. DELAFOSSE A., BENGALY Z., DUVALLET G., 1996. Utilisation du test Elisa de détection des antigènes circulants de trypanosomes dans le cadre d'un suivi épidémiologique dans la zone de Sidéradougou, Burkina Faso. Revue Elev. Méd. vét. Pays trop., 49 : 32-37.

3. DESQUESNES M., BENGALY Z., DIA, M.L., 2003. Evaluation de la persistance des anticorps détectés par Elisa-indirect Trypanosoma vivax après traitement trypanocide chez des bovins naturellement infectés. Revue Elev. Méd. vét. Pays trop., 56 : 141-144.

4. DUVAlLeT G., DE LA ROCQUE S., REIFENBERG J.M., SOLANO P., LEFRANÇOIS T., MICHEL J.F., BENGALY Z., SIDIBE I., CUISANCE D., CUNY G., 1999. Review of the molecular tools for the understanding of the epidemiology of animal trypanosomosis in West Africa. Mem Inst. Oswaldo Cruz, 94: 245-248.

5. LUCKINS A.G., 1977. Detection of antibodies in trypanosome-infected cattle by means of a microplate enzyme-linked immunosorbent assay. Trop. Anim. Health Prod., 9: 53-62.

6. NANTULYA V.M., LINDQVIST K.J., 1989. Antigen-detection enzyme immunoassays for the diagnosis of Trypanosoma vivax, T. congolense and T. brucei infections in livestock. Trop. Med. Parasit., 40: 267-271.

Accepté le 13.02.2010

\section{Summary}

Barry A.M., Roger F., Diallo M.B., Geerts S. Evaluation of Cattle Trypanosomosis Seroprevalence in Guinea

An evaluation of trypanosomosis prevalence was carried out in Guinea on sera originating from the serum bank of the veterinary laboratory of the National Livestock Services. Nine hundred and twenty-eight sera sampled on N'dama cattle were randomly selected and analyzed for antibody and antigen detection of Trypanosoma spp. Results obtained in the first test revealed Trypanosoma spp. antibodies in $67 \%$ of the cattle, with rates of $72 \%$ in the High Guinea area, $68 \%$ in Lower Guinea, $63 \%$ in Forest Guinea, and $62 \%$ in Middle Guinea. Among the animals, $69 \%$ of the females were infected or had been in contact with the infectious agent, against $62 \%$ of the males. The animals were infected regardless of their age. Infection rates were $71,43,61,63,59$, and $74 \%$ in cattle under one year of age, 1-2 years old, 2-3 years old, 3-4 years old, 4-5 years old, over 5 years old, respectively. The second test showed the presence of antigens against T. brucei $(16 \%$ of the sera), T. congolense (11\%) and T. vivax ( $2 \%)$. The distribution of the results of both tests showed that $2 \%$ of the animals were positive for one, two or three antigens without the presence of antibodies, $17 \%$ were positive for both antigen(s) and antibodies, and $50 \%$ were positive for antibodies only.

Keywords: Cattle - Antigen - Antibody - Trypanosoma Immunodiagnosis - Guinea.

\section{Resumen}

Barry A.M., Roger F., Diallo M.B., Geerts S. Evaluación de la seroprevalencia de la tripanosomosis bovina en Guinea

Se efectuó una evaluación de la prevalencia de la tripanosomosis en Guinea, en sueros provenientes de la seroteca del laboratorio de diagnóstico veterinario de la Direction Nationale de I'Elevage. Se seleccionaron aleatoriamente novecientos veintiocho sueros procedentes de bovinos N'dama y se analizaron mediante las pruebas de detección de anticuerpos de Trypanosoma spp. y de antígeno. Los resultados obtenidos por la primera prueba revelaron la presencia de anticuerpos de Trypanosoma spp. en el $67 \%$ de los bovinos, con tasas del $72 \%$ en la región de la Guinea Alta, del $68 \%$ en la Guinea Baja, del $63 \%$ en la Guinea Forestal y del $62 \%$ en la Guinea Media. Dentro de los animales, el $69 \%$ de las hembras estaba infectado o había estado en contacto con el agente infeccioso, frente al $62 \%$ de los machos. Los animales estaban infectados con independencia de la edad. Las proporciones de infecciones fueron del 71, 43, $61,63,59$ y $74 \%$ en los bovinos con edades inferiores a 1 año, de 1 a 2 años, de 2 a 3 años, de 3 a 4 años, de 4 a 5 años y de más de 5 años, respectivamente. La segunda prueba reveló la presencia de antígenos de T. brucei (16\% de los sueros), de T. congolense $(11 \%)$ y de T. vivax (2\%). La distribución y combinación de los resultados mostró que el $2 \%$ de los animales eran positivos a uno, dos o tres antígenos sin rastro de anticuerpos; el $17 \%$ de los animales resultaron positivos a los antígenos y a los anticuerpos y la mitad de los animales sólo tenían anticuerpos.

Palabras clave: Ganado bovino - Antígeno - Anticuerpo Trypanosoma - Inmunodiagnóstico - Guinea. 